\title{
Viv: Multiscale Visualization of High-Resolution Multiplexed Bioimaging Data on the Web
}

Trevor Manz ${ }^{1}$, Ilan Gold ${ }^{1}$, Nathan Heath Patterson², Chuck McCallum², Mark S Keller ${ }^{1}$, Bruce W Herr II ${ }^{3}$, Katy Börner ${ }^{3}$, Jeffrey M Spraggins ${ }^{2}$, Nils Gehlenborg ${ }^{1, *}$

${ }^{1}$ Harvard Medical School, Boston, MA, USA

${ }^{2}$ Vanderbilt University, Nashville, TN, USA

${ }^{3}$ Indiana University, Bloomington, IN, USA

*Corresponding author: nils@hms.harvard.edu 


\section{Manuscript (788 words)}

Advances in highly multiplexed imaging ${ }^{1-4}$ have enabled the comprehensive profiling of complex tissues in healthy and diseased states, facilitating the study of fundamental biology and human disease at spatially-resolved, subcellular resolution ${ }^{5,6}$. While the rapid innovation of biological imaging poses significant scientific value, the proliferation of technologies without unification of interoperable standards have created challenges limiting analysis and sharing of results. The adoption of community-designed next-generation file formats (NGFF) is a proposed solution to promote bioimaging interoperability at scale $^{7}$. Here we introduce Viv - an open-source bioimaging visualization library which supports OME-TIFF $^{8}$ and OME-NGFF $^{7}$ directly on the web. Viv addresses a critical limitation of most web-based bioimaging viewers by removing a dependency on server-side rendering, offering a flexible toolkit for browsing multi-terabyte datasets on both mobile and desktop devices - without software installation.

Viv functions more similarly to popular desktop bioimaging applications than corresponding web alternatives (see Supplemental Note 1). Most web viewers require the pre-translation of large binary data files into rendered images (PNG or JPEG) to display in a browser client. Two existing approaches perform this step (server-side rendering) but differ with regard to when rendering occurs and how much of the binary data is transformed at one time (see Figure 1). The offline option performs all rendering before an application is deployed to users, meaning all channel groupings and data transformations are fixed and cannot be adjusted via the user interface. The online option supports on-demand, user-defined rendering but introduces latency when exploring data transformations and requires active maintenance of complex server infrastructure. Neither approach provides a flexible solution to directly view datasets saved in open formats from large scale public data repositories, and the transient data representation introduced by server-side rendering inhibits interoperability with other visualization and analysis software.

Viv implements purely client-side rendering to decouple the browser from the server and still offer the flexibility of on-demand multi-channel rendering. Existing web viewers also leverage GPU-accelerated rendering but are generally tailored towards single-channel volumetric datasets and crucially lack the ability to reuse and compose features for existing or novel applications $^{9,10}$. In contrast, Viv's modularity allows core functionality to be repurposed and extended. The library consists of two primary components: (1) modules for lazily loading OME-TIFF and OME-NGFF data formats and (2) configurable GPU programs that render full bit-depth primary data on user devices. 


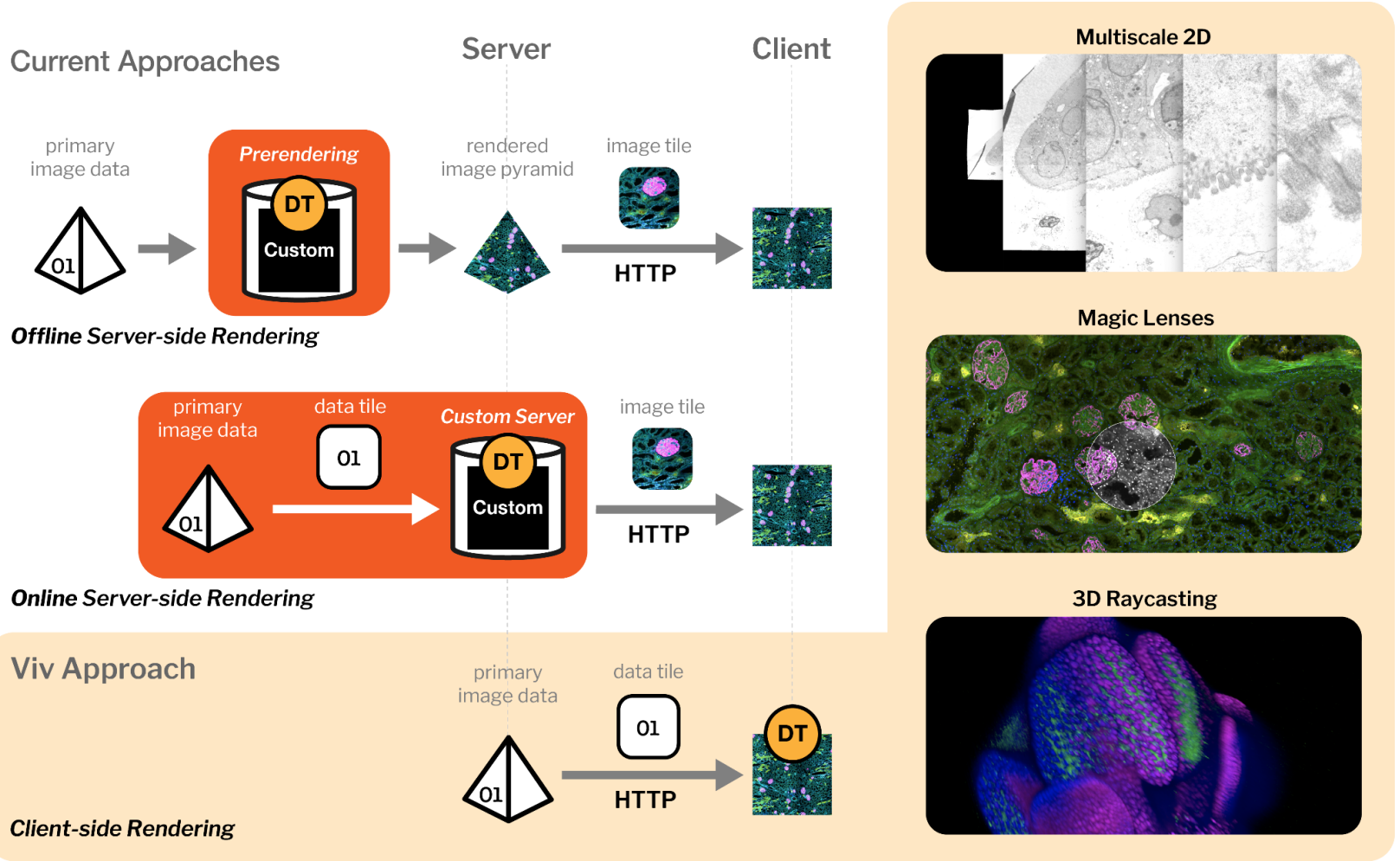

Figure 1. Overview of data flow and rendering approaches for web-based bioimage data visualization and Viv features. DT indicates the location where data is transformed into an image. The right column displays a subset of Viv's flexible client-side rendering, including multiscale $2 D$ pyramids, magic lenses, and $3 D$ volumes via raycasting.

The data loading component of Viv is responsible for fetching and decoding atomic "chunks" of compressed channel data from binary files via HTTP. We targeted both OME-TIFF and OME-NGFF to suit the diverse needs of the bioimaging community and promote the use of interoperable open standards. While OME-TIFF is more ubiquitous, its binary layout and metadata model are too restrictive to efficiently represent large volumes and datasets with high dimensionality. OME-NGFF is designed to address these limitations and is natively accessible from the cloud due to the underlying multidimensional Zarr format ${ }^{11}$. Zarr provides direct access to individual chunks whereas IIFF requires seeking to access chunks from different planes. introducing long latencies when reading datasets with large $C, T$, or $Z$ dimensions ${ }^{7}$. Similar to approaches popularized in genomics ${ }^{12}$, we propose indexing OME-TIFF to achieve more efficient random access. Indexed OME-TIFF improves access times and critically is implemented as an optional, complementary extension to OME-TIFF (see Supplementary Note 2).

Viv's image layers coordinate fetching data chunks and GPU-accelerated rendering. All rendering occurs on the client GPU, allowing continuous and immediate updates of properties such as color mapping, opacity, channel visibility, and affine coordinate transformation without additional data transfer. Library users can modify rendering with custom WebGL shaders, a 
feature we utilized to implement magic lenses ${ }^{13}$ that apply local data transformations, e.g., rescaling brightness or filtering specific channels.

By moving all rendering to the browser, Viv does not rely on a server like previous web viewers for highly multiplex and multiscale images, making it flexible to embed within a variety of applications (see Supplementary Note 3). We integrated Viv into Jupyter Notebooks ${ }^{14}$ via ImJoy ${ }^{15}$ to enable a remote human-in-the-loop multimodal image registration workflow. Viv also underlies the imaging component of the Vitessce single-cell visualization framework ${ }^{16}$, demonstrating its ability to integrate with and power other modular tools that include imaging modalities. Finally, we developed Avivator (http://avivator.gehlenborglab.org), a standalone image viewer that showcases Viv's rich feature set.

Due to its extensibility and minimal deployment requirements, Viv offers an effective toolkit to build a wide range of reusable bioimaging applications. It is best suited for displaying 2D visualizations of highly multiplex and multiscale datasets, although 3D visualizations via raycasting are also supported. We do not expect Viv to replace existing web-based viewers but instead shepherd a new generation of web-based visualization and analysis tools to complement desktop software also built around interoperable standards.

\section{Acknowledgments}

Viv was developed with funding from the National Institutes of Health (OT2OD026677, T15LM007092, T32HG002295), the National Science Foundation (DGE1745303), and the Harvard Stem Cell Institute (CF-0014-17-03). The use cases in this manuscript were supported with additional funding from the National Institutes of Health (U54DK120058, 2P41GM103391, OT2OD026671) and National Science Foundation (CBET1828299). We would also like to thank members of the OME community for their guidance as well as Mark deCaestecker, Elizabeth Neumann, and Maya Brewer from Vanderbilt University and Vanderbilt University Medical Center for their efforts generating the imaging mass spectrometry and microscopy data highlighted in the Use Cases.

\section{Competing Interests}

N.G. is a co-founder and equity owner of Datavisyn.

\section{Code Availability}

Viv is open source and available under an MIT license at https://github.com/hms-dbmi/viv and at https://www.npmjs.com/package/@hms-dbmi/viv. 


\section{References}

1. Alexandrov, T. Spatial Metabolomics and Imaging Mass Spectrometry in the Age of Artificial Intelligence. Annu. Rev. Biomed. Data Sci. 3, 61-87 (2020).

2. Goltsev, Y. et al. Deep Profiling of Mouse Splenic Architecture with CODEX Multiplexed Imaging. Cell 174, 968-981.e15 (2018).

3. Saka, S. K. et al. Immuno-SABER enables highly multiplexed and amplified protein imaging in tissues. Nat. Biotechnol. 37, 1080-1090 (2019).

4. Lin, J.-R. et al. Highly multiplexed immunofluorescence imaging of human tissues and tumors using t-CyCIF and conventional optical microscopes. Elife 7, (2018).

5. Consortium, H. \& HuBMAP Consortium. The human body at cellular resolution: the NIH Human Biomolecular Atlas Program. Nature vol. 574 187-192 (2019).

6. Rozenblatt-Rosen, O. et al. The Human Tumor Atlas Network: Charting Tumor Transitions across Space and Time at Single-Cell Resolution. Cell 181, 236-249 (2020).

7. Moore, J. et al. OME-NGFF: a next-generation file format for expanding bioimaging data-access strategies. Nat. Methods 1-3 (2021).

8. Goldberg, I. G. et al. The Open Microscopy Environment (OME) Data Model and XML file: open tools for informatics and quantitative analysis in biological imaging. Genome Biol. 6, R47 (2005).

9. Saalfeld, S., Cardona, A., Hartenstein, V. \& Tomancak, P. CATMAID: collaborative annotation toolkit for massive amounts of image data. Bioinformatics 25, 1984-1986 (2009).

10. Boergens, K. M. et al. webKnossos: efficient online 3D data annotation for connectomics. Nat. Methods 14, 691-694 (2017). 
11. Miles, A. et al. zarr-developers/zarr-python: v2.4.0. (2020). doi:10.5281/zenodo.3773450.

12. Li, H. Tabix: fast retrieval of sequence features from generic TAB-delimited files. Bioinformatics 27, 718-719 (2011).

13. Bier, E. A., Stone, M. C., Pier, K., Buxton, W. \& DeRose, T. D. Toolglass and magic lenses: the see-through interface. in Proceedings of the 20th annual conference on Computer graphics and interactive techniques 73-80 (Association for Computing Machinery, 1993).

14. Kluyver, T. et al. Jupyter Notebooks-a publishing format for reproducible computational workflows. in ELPUB 87-90 (2016).

15. Ouyang, W., Mueller, F., Hjelmare, M., Lundberg, E. \& Zimmer, C. ImJoy: an open-source computational platform for the deep learning era. Nat. Methods 16, 1199-1200 (2019).

16. Keller, M. S. et al. Vitessce: a framework for integrative visualization of multi-modal and spatially-resolved single-cell data. OSF Preprints (2021) doi:10.31219/osf.io/y8thv. 


\section{Supplementary Note 1}

\section{Motivation}

Visualization of highly multiplexed, high-resolution tissue datasets on the web relies on client software that displays server-side rendered images ${ }^{1,2}$ rather than rendering data in the client. The JavaScript clients for many of these applications, such as Minerva ${ }^{2}$, Facetto ${ }^{3}$, Human Protein Atlas Microscopy Viewer ${ }^{4}$, OMERO iViewer ${ }^{5}$, Digital Slide Archive ${ }^{6}$, and Cytomine ${ }^{7}$ are based on tools like OpenSeadragon ${ }^{8}$ or OpenLayers ${ }^{9}$, which are popular web-based viewers for zoomable images. The viewers load image tiles encoded in RGB/A data formats that are natively supported by web browsers (e.g., PNG, JPEG). Therefore primary high-resolution datasets, including those stored in open standard formats, must first be rendered to image tiles in order to use these tools. Image tiles can be rendered dynamically, as with client-server models like $\mathrm{OMERO}^{5}$, or statically, where each level of the multi-resolution representation (pyramidal data) is pre-rendered as a nested directory of PNG or JPEG files (see Figure 1).

Whole-slide datasets derived from bright-field assays such as Hematoxylin and Eosin (H\&E) stains or Periodic acid-Schiff (PAS) stains only contain red, green, and blue (RGB) channels and do not require additional data transformations to render. This means current web technologies are sufficient for viewing these datasets, despite the overhead of conversion to browser-friendly image tiles, since only zooming and panning interactions are necessary for exploration.

Datasets produced by multiplexed imaging methods ${ }^{10-13}$, however, are instead most often stored as multidimensional stacks of 2D planes where each plane-or channel-typically represents a chemical signal, such as a fluorophore bound to an antibody in immunofluorescence. Images derived from non-targeted methods like imaging mass spectrometry ${ }^{14}$ (IMS) are stored similarly with a deep $\mathrm{m} / \mathrm{Z}$ dimension. Multidimensional stacks (including T, C, Z dimensions) may contain over a hundred 16- or 32-bit grayscale planes that must be pseudo-colorized for visualization, and it is desirable to view each channel separately since the specificity of the acquisition methods is high. Therefore, effective interactive analysis of these datasets require both zoom and pan interactions as well as rapid switching between groups of channels via sophisticated rendering.

\section{Multi-Channel Rendering}

Multi-channel rendering involves the blending of data from separate channels into a single image, allowing users to view isolated signals or blended composites during analysis. Individual channel data transformations are applied per pixel to generate a final image. Efficient multi-channel rendering is especially important to oncologists and pathologists who rely on seamless visual experience when making a diagnosis ${ }^{3}$. Multi-channel color mapping is increasingly important in modern cell-based image analysis, where many channel combinations 
are possible with limited overlap, and channels may be grouped according to some biologically relevant criteria ${ }^{3}$.

Desktop applications are the current gold standard for interactive analysis and support many types of rendering. In contrast to existing web-based viewers, desktop-oriented programs make use of low-latency connections to primary data and leverage graphics cards for rapid rendering. Specific software and hardware requirements, however, restrict the availability of these tools to a wider scientific audience, and the coupling of data storage and computational environment limit the user experience when viewing remote data, if supported, over low-latency connections.

Most existing JavaScript tools for viewing multiscale images do not implement multi-channel rendering, and instead tightly coupled client-server architectures are utilized to enable interactive analysis of highly multiplexed datasets on the web ${ }^{3,15}$. It is important to note that this approach strictly avoids sending raw data to the client, which has the benefit of smaller data transfer per request, but ultimately the client is totally dependent on the server for information. Sampling many rendering settings within a server-rendered viewer incurs a server-round-trip and additional data transfer per configuration, whereas loading raw data on the client is expensive upfront but subsequent re-renders are instantaneous and do not require additional data transfer.

Offline server-side rendering is accomplished by applying a set of data transformations to primary imaging data prior to configuring a web server, yielding a unique rendered copy of the original dataset (directory hierarchy of JPEG or PNG image tiles) for each combination of channel groupings and data transformations. Although this approach is simple to deploy, it prevents end users from on-demand visual exploration since data transformations and channel groupings are fixed and only determined offline. Consequently, web viewers of this type are tailored towards explanatory visualization where users are guided through a narrative that accompanies the pre-rendered image pyramids ${ }^{2}$.

In online server-side rendering, users define channel groupings and data transformations via a web user interface, and then a server applies the desired transformations on-the-fly and renders image tiles to send back to the client. While this approach has been implemented successfully, it requires substantial maintenance and the tight coupling of client and server inhibits the use of either in isolation. User experience also degrades when the server is at capacity or over a slow network connection since adjusting individual channel transformations, sampling channel groupings, or toggling channel visibility requires the client to await a new server-rendering. In contrast, identical user interactions in analogous desktop software yield low-latency, continuous updates since graphics cards are exploited to quickly apply new data transformations to the same in-memory data.

WebGL (Web Graphics Library) allows similar access to the GPU as desktop software but has yet to be applied to enable multi-channel rendering of high-resolution, multiplexed image data on the web. Existing WebGL-based viewers showcase the potential for complex browser-based rendering but are primarily designed for single-channel volumetric datasets (typically 
lossy-compressed, 8-bit dense arrays), rely on data to fit in-memory for visualization, or still use custom server implementations ${ }^{16-19}$. Additionally, these viewers are fully-integrated applications and as such do not provide reusable components for building or extending existing interactive bioimaging visualizations.

\begin{tabular}{|c|c|c|}
\hline Name & Description & URL \\
\hline WebGL & $\begin{array}{l}\text { Low-level JavaScript API for rendering } \\
\text { high-performance interactive graphics. }\end{array}$ & https://www.khronos.org/webgl/ \\
\hline WebAssembly & $\begin{array}{l}\text { Portable binary-code format that is } \\
\text { executable in modern web browsers. }\end{array}$ & https://www.w3.org/TR/wasm-core-1/ \\
\hline WebWorkers & $\begin{array}{l}\text { Web API to run scripts in a separate thread } \\
\text { from the main execution thread. }\end{array}$ & https://www.w3.org/TR/workers/ \\
\hline geotiff.js & $\begin{array}{l}\text { A JavaScript library to parse TIFF files for } \\
\text { web-based visualization. }\end{array}$ & https://geotiffjs.github.io/ \\
\hline Zarr.js & A TypeScript implementation of Zarr ${ }^{20}$. & https://github.com/gzuidhof/zarr.js/ \\
\hline Deck.gl $\left.\right|^{21}$ & $\begin{array}{l}\text { WebGL-powered framework for } \\
\text { high-performance visualization. }\end{array}$ & https://deck.gl/ \\
\hline Bio-Formats ${ }^{15}$ & $\begin{array}{l}\text { Software tool suite for reading and writing } \\
\text { image data using standardized, open } \\
\text { formats. }\end{array}$ & https://www.openmicroscopy.org/bio-formats/ \\
\hline bioformats2raw & $\begin{array}{l}\text { Java application to convert proprietary } \\
\text { image file formats to intermediate N5/Zarr } \\
\text { structure. }\end{array}$ & https://github.com/glencoesoftware/bioformats2raw \\
\hline raw2ometiff & $\begin{array}{l}\text { Java application to convert outputs of } \\
\text { bioformats2raw to an OME-TIFF pyramid. }\end{array}$ & https://github.com/glencoesoftware/raw2ometiff \\
\hline Blosc $^{22}$ & $\begin{array}{l}\text { Lossless compression library used by } \\
\text { Zarr }^{20} \text {. }\end{array}$ & https://github.com/Blosc/c-blosc \\
\hline
\end{tabular}

Supplementary Table 1. Software libraries and web technologies used by Viv.

\section{Software Architecture}

Viv is a JavaScript library that provides GPU-accelerated multi-channel rendering of high-resolution multiplexed images directly in the web browser. It is implemented in the TypeScript programming language (https://www.typescriptlang.org), allowing ease of use in other JavaScript and TypeScript projects. As a design philosophy, we built Viv on top of open standard image formats created by the Open Microscopy Environment (OME), OME-TIFF ${ }^{23}$ and

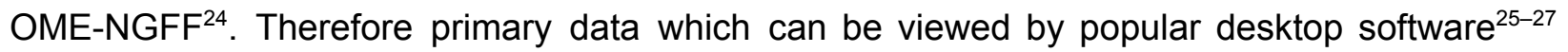
may also be viewed directly by Viv without converting to a transient browser-friendly format.

Due to its modular architecture, Viv is embeddable and can be deployed to support visualization for exploration and explanation in a wide range of different settings. Since Viv operates on multi-resolution data formats, the rendering performance is not affected by the size of the 
images. We designed Viv as a modular JavaScript library using modern web technologies (see Supplementary Table 1) to support dynamic fetching, decoding, and rendering of pyramidal multiplex datasets. The data loading component of Viv is built on the geotiff.js and zarr.js libraries, to which we contributed additional features to support efficient data chunk retrieval. Viv exposes its rendering functionality as reusable Deck.g ${ }^{21}$ layers, allowing the composition of multiple image sources in a single interactive view. The Deck.gl library has no dependencies on web user interface frameworks and can be used in any JavaScript application, enabling Viv to be incorporated into existing client software with little overhead. Finally, Viv offers several custom high-level React components that handle complex rendering and interactivity, such as overview and detail and multiple linked views.

\section{Data Preparation}

Bio-Formats ${ }^{15}$ is a software tool for reading proprietary microscopy image data and metadata using standardized, open formats. It provides the ability to translate over 150 file formats and associated metadata to the OME data model. OME-TIFF has been available for over a decade and is a common format for sharing imaging data. It is recommended and used by the Image Data Resource ${ }^{28}$ and various consortia such as Human BioMolecular Atlas Program ${ }^{29}$ and the 4D Nucleome Consortium ${ }^{30}$. OME-NGFF is a complementary open format that is designed to address fundamental limitations of OME-TIFF at scale. The format is under active collaborative development by the OME community as a cloud-friendly solution to provide flexible storage of multidimensional datasets from established and emerging imaging assays.

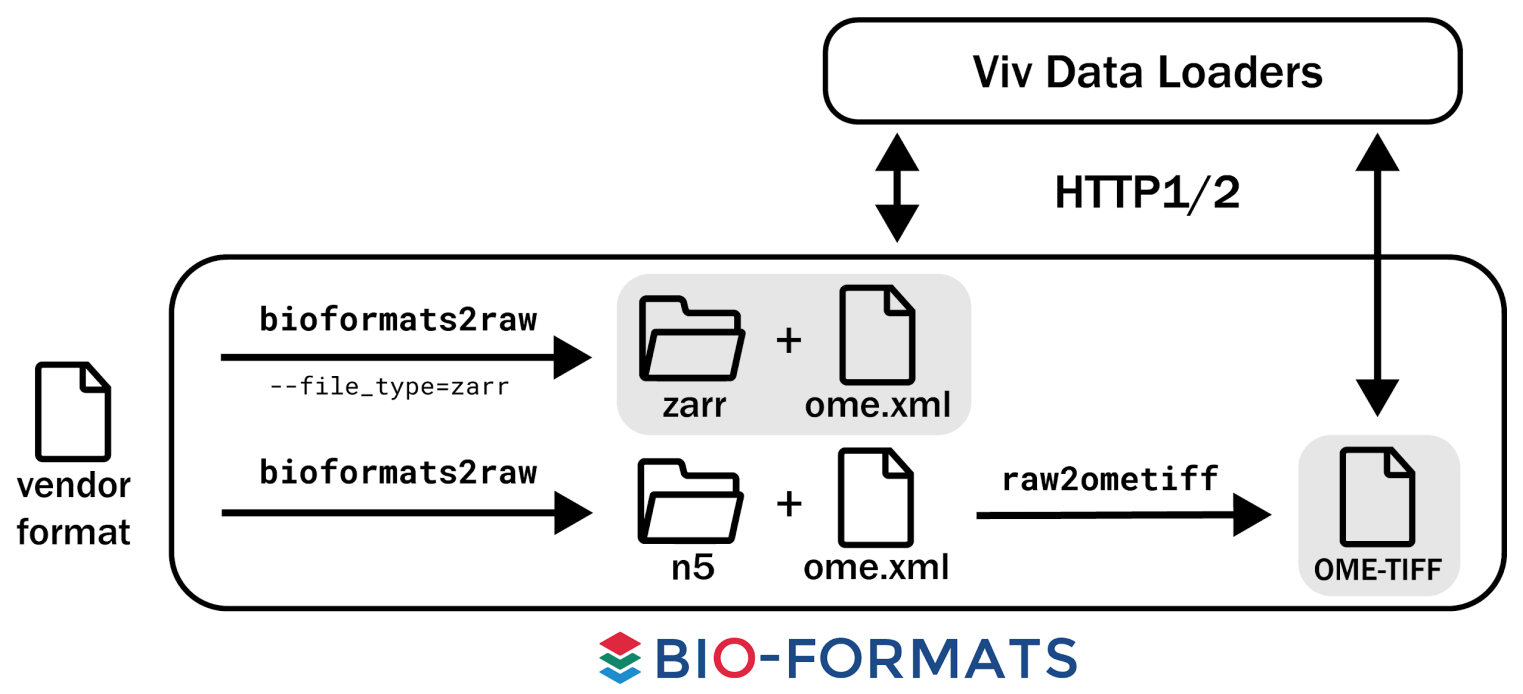

Supplementary Figure 1. Viv Bio-Formats compatibility. The Bio-Formats command-line tools are used sequentially to generate OME-NGFF and OME-TIFF images. The bioformats2raw creates a "raw" intermediate Zarr or N5 which is subsequently converted to an OME-TIFF via raw2ometiff. Viv's data loader utilities are compatible with both OME-NGFF and OME-TIFF formats. 
Viv supports viewing both OME-NGFF and OME-TIFF directly via HTTP (Supplementary Figure 1). Writing OME-NGFF and OME-TIFF can be accomplished using the Bio-Formats command-line suite. The bioformats2raw utility (https://github.com/glencoesoftware/bioformats2raw) is responsible for converting proprietary image formats to Zarr, generating pyramidal levels from large resolution planes if not available. The Bio-Formats pipeline subsequently converts this representation to OME-TIFF via raw2ometiff (https://github.com/glencoesoftware/raw2ometiff).

Once created, Viv can access these data on-demand via HTTP. A local web-server is sufficient for viewing datasets locally, and full web applications may be deployed by uploading the same datasets to a commodity web-server or commercial cloud object storage (e.g., Amazon S3, Google Cloud Storage, Microsoft Azure Blob).

\section{Data Loading}

Viv's data loaders provide a consistent interface for retrieving data tiles from arbitrary sources. Data loaders fetch individual compressed chunks from OME-TIFF or OME-NGFF via HTTP, and subsequently decompress these data for rendering. Custom loaders may be implemented to support additional data sources.

An important component of Viv's data loaders is the support for Zarr ${ }^{20}$, the binary format underlying OME-NGFF. Zarr is an open-source format for the storage of chunked, compressed, multidimensional arrays. The original implementation of Zarr is written in Python, but its popularity has led to implementations in several languages ( +++ , Java, Julia, JavaScript). The underlying compressed chunk data and array metadata can be saved in any key-value store, most commonly a local file directory or cloud object storage. This flexibility allows the configuration of custom storage for application-specific needs, meaning various backends can be utilized to support visualizations with Viv. For example, our Viv-based Vizarr viewer implements a custom Zarr interface to securely transfer data from a Python backend via the ImJoy ${ }^{31}$ Remote Procedure Call (https://github.com/imjoy-team/imjoy-rpc).

Data loading from OME-TIFF files is handled through HTTP byte-range requests. OME-TIFF defines its multi-resolution representation via TIFF Sub-Image File Directories (SubIFDs) (https://www.adobe.io/content/dam/udp/en/open/standards/tiff/TIFFPM6.pdf) which are accessed on the client to load specific sub-resolutions. Simply reading OME-TIFF via HTTP is limited, however, due to TIFF's linear binary layout which was designed for local filesystems. We propose the use of a complementary pre-computed index to avoid seeking on the client and improve the efficiency of random chunk access (see Supplementary Note 2).

Data retrieval for all loaders is done in an asynchronous event loop so that multiple compressed chunks can be fetched concurrently. Web Workers are used to perform chunk decoding on separate threads, providing parallelism and freeing the user interface to remain responsive to user interactions. Popular image compression methods have been ported to JavaScript previously, but desktop software typically relies on libraries written in low-level languages like C 
or $\mathrm{C}++$ for performing binary decompression. WebAssembly enables the reuse of these same libraries in a web browser with similar performance. We compiled the modern high-performance compressor Blosc ${ }^{22}$ (Zarr default), Lempel-Ziv-Welch (LZW) ${ }^{32}$ (OME-TIFF default), and the popular Zstd and LZ4 algorithms to WebAssembly to support rapid and flexible decoding.

\section{Rendering Component}

The multi-channel rendering component of Viv is implemented as custom Deck.gl layers. Deck.gl is a WebGL-powered framework for exploratory visualization of large, spatial datasets. A layer is a core concept of Deck.gl. It describes a packaged visualization type that combines a collection of data and methods to render these data in a shared coordinate system. Interactive Deck.gl visualizations can be constructed by composing layers with others (points, polygons, text annotations, etc.), enabling highly customizable and efficient rendering of complex scenes.

Viv layers control what is rendered when a user interacts with the WebGL canvas. The declarative layer API provides the ability to define specific channel selections from a multidimensional source as well as desired data transformations per channel. Additionally, affine transformations are supported per layer via a $4 \times 4$ transformation matrix. These parameters can be updated within a reactive paradigm, enabling GPU-accelerated rendering across modern web frameworks and user interfaces. Each Viv layer uses a data loader to retrieve individual chunks for the corresponding channel selections as a user zooms and pans in the coordinate space. Chunks are fetched and decoded by the loaders and then loaded on the GPU, where shaders apply the user-defined data transformations. Once the data have been retrieved for a particular region, changes to desired transformations (contrast limits, opacity, color mapping, visibility) simply re-render using the previously loaded data. This creates a low-latency user experience when exploring channel combinations and data transformations.

To enable the rapid exploration of spatial distributions and correlations between channels, Viv provides a reactive API for blending the data from different channels into a single image layer. Data chunks contain the pixel intensities for individual channels, and once bound to the GPU, data transfer functions are applied one of two ways:

1. Additive Blending. Each channel is assigned an RGB (or equivalently, HSV) color value, defining a linear color transfer function that maps black to the minimum value and the color to the maximum value per channel. The contributions of individual channels are then additively blended into a single RGBA image following min-max normalization. The respective contrast limits, as well as the colors for each transfer function, are exposed via the Viv Layer API. This type of additive blending ensures non-overlapping colors for up to three channels when using pure red, green, and blue. Viv currently supports up to six concurrently rendered channels per image, which is useful when viewing additional channels that have little to no spatial overlap.

2. Additive Color Mapping. The second option is to use a single transfer function that maps the combined channel intensities to a colormap such as Viridis or Magma. Each channel intensity is min-max normalized to $0-1$, and all normalized intensities are 
summed. Sum is then used as the input for a transfer function. This method is similar to the lookup tables supported by OMERO iViewer ${ }^{5}$. We use glslify ${ }^{33}$ to inject transfer functions into the Viv shaders which scale intensity values to RGB colors.

When multiple images are loaded into Viv, alpha compositing between layers is supported. Additionally, rendering is completely flexible in Viv, and library users are able to implement custom shaders to modify the builtin behavior described above.

\section{Viv API}

The Viv API comprises the following major elements. Details are available in the documentation (http://viv.gehlenborglab.org).

1. Viewers. Drop-in React components that expose interfaces for developers to supply controllers for the various rendering settings. Handle complex rendering and interactivity, such as overview and detail and multiple linked views.

2. Views. Building blocks for supporting viewers with multiple views, like side-by-side or picture-in-picture. A view is a stateful object defined by a particular zoom level and bounding box.

3. Layers. Control what spatial regions and channels to render in each view, along with what data transformations to apply per channel. Viv provides MultiscaleImageLayer and ImageLayer layers that support rendering pyramidal and nonpyramidal images, respectively. The VolumeLayer supports rendering 3D views via ray casting.

4. Loaders. Shared interface for accessing the metadata and channel data from OME-TIFF and OME-NGFF via HTTP.

\section{Supplementary Note 2}

\section{Random Chunk Access Latency for OME-NGFF, OME-TIFF, and Indexed OME-TIFF}

Viv loads atomic chunks directly from OME-TIFF and OME-NGFF via HTTP, contrasting most existing web clients which rely on server-rendered image tiles. The underlying binary layout for each format, TIFF and Zarr respectively, therefore govern the efficiency of random access. Zarr provides direct access to individual chunks whereas TIFF requires seeking to find chunks from separate 2D planes. This difference enables Zarr to achieve comparable throughput on local filesystems and remote servers, while throughout suffers substantially in the absence of low-latency local disk access for TIFF ${ }^{24}$. 
Similar to approaches popularized in genomics ${ }^{34,35}$, we propose indexing OME-TIFF to improve random access efficiency. We developed a python command line utility (and website) to generate an OME-TIFF index (JSON) containing the corresponding byte-offsets for each TIFF Image File Directory (IFD) (https://github.com/hms-dbmi/generate-tiff-offsets). Viv utilizes the byte-offsets to skip the otherwise required step of linearly seeking the series of IFDs, providing more direct access to individual chunks. An OME-TIFF contains one IFD per full-resolution 2D plane, meaning the total number of indexed byte-offsets is equal to $C \times T \times Z$ and independent of the number of pyramidal sub-resolutions.

To illustrate the differences in read efficiency, we compare the latency of random chunk access via HTTP between OME-NGFF, OME-TIFF, and OME-TIFF with an IFD index (Indexed OME-TIFF). We measured the reading time of individual chunks from two light sheet fluorescence microscopy datasets ${ }^{36}, 2 \mathrm{D}$ multi-channel $(X=1920, Y=1920, Z=1, C=4, T=1)$ and 3D multi-channel $(X=1920, Y=1920, Z=259, C=4, T=1)$, converted to these formats. All code is available for downloading and converting datasets from the $\mathrm{IDR}^{28}$ and running the benchmark (https://github.com/ome/bioimage-latency-benchmark/tree/main/js).

We observed that access speeds for OME-NGFF outperform OME-TIFF with and without an index, but importantly Indexed OME-TIFF substantially reduces OME-TIFF chunk access latencies (see Supplementary Figure 2). For OME-TIFF, access times for individual chunks are related to the corresponding plane's IFD position in the monolithic file, showing longer latencies for chunks that require more seeking. This relationship is not observed in OME-NGFF or Indexed OME-TIFF where chunk access is direct and avoids seeking.

Together these results suggest Indexed OME-TIFF is a pragmatic solution to progressively enable efficient random access to OME-TIFF. The relative access speed improvement of Indexed OME-TIFF over OME-TIFF is dependent on the dataset dimensionality. For multi-channel 2D datasets, there is marginal benefit of Indexed OME-TIFF over OME-TIFF since seeking is limited to a few planes in total. However, multidimensional OME-TIFFs increasingly benefit from indexing as $Z$, $T$, or $C$ dimensions grow in size, improving access speeds by nearly two orders of magnitude for the multi-channel 3D dataset in our benchmark. 

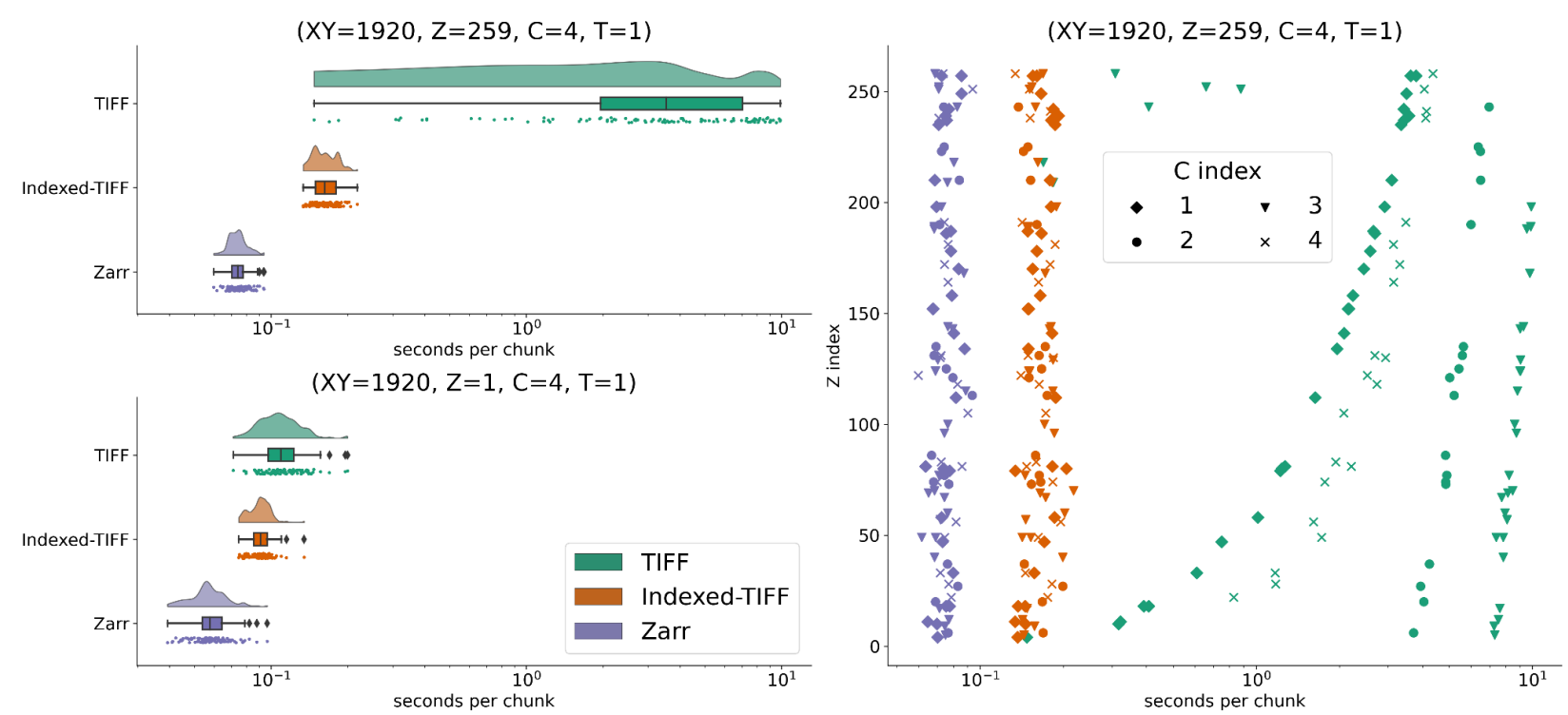

Supplementary Figure 2. Indexed OME-TIFF improves OME-TIFF chunk retrieval times within an order of magnitude of OME-NGFF. Random sampling of 100 uncompressed chunks from multi-channel 2D and 3D datasets converted to OME-NGFF ("Zarr", purple), OME-TIFF ("TIFF", green), and Indexed OME-TIFF ("Indexed-TIFF", orange). Chunks are loaded via HTTP from a NGINX web server with default configuration. The rain cloud plots on the left show that as dataset dimensions increase in size, latencies increase substantially for OME-TIFF due to additional seeking. The scatter plot on the right shows that access speeds for individual chunks are related to the corresponding $Z$ and $C$ coordinates in OME-TIFF but not OME-NGFF or Indexed OME-TIFF.

Viv is currently the only client which supports Indexed OME-TIFF, but clients implemented in other programming languages could similarly reuse the IFD index to provide efficient chunk access. A noted downside of OME-NGFF is the increase of on-disk complexity as file sizes increase, potentially limiting copying and sharing of datasets ${ }^{24}$. Indexed OME-TIFF can be viewed as a compromise between OME-NGFF and OME-TIFF, allowing better portability compared to OME-NGFF with improved chunk access speed compared to OME-TIFF. This method could potentially be extended to other more modern monolithic formats, such as HDF5, to offer a similar compromise of portability and remote read efficiency for datasets where the OME-TIFF binary layout and metadata model are too restrictive. 


\section{Supplementary Note 3}

\section{Use Cases}

\section{Image Registration Workflow}

Image registration is a critical task in biomedical image analysis. Accurate alignment of datasets captured from different modalities is imperative for integrating histological information with molecular information to produce high-quality, comprehensive tissue profiles. The ability to quickly configure, test, and compare different registration methods is desirable during the alignment process but often requires large amounts of memory and/or CPUs to be efficient. Therefore institutional or cloud computational resources are attractive compared to personal computers when working with multiple high-resolution images, especially when the data are already located on the same high-performance servers. However, working with data remotely currently requires leaving the computational environment used for creating the alignment (e.g., Jupyter Notebook) to assess the quality of registration, slowing the ability to iterate different methods while optimizing the registration.

We created the OME-NGFF viewer, Vizarr (https://github.com/hms-dbmi/vizarr), using Viv to enhance an image registration workflow and enable interactive viewing of multiple multiscale images from within a Jupyter Notebook. The registration example diagrammed in Supplementary Figure 3 details this workflow, displaying an overview of the registration of multiple image modalities using the wsireg library (https://github.com/NHPatterson/wsireg) and remote viewing of subsequent alignments all within the same Jupyter Notebook. The registration approach $^{37,38}$ uses an autofluorescence (AF) microscopy intermediary captured on two serial sections prior to other imaging experiments (e.g., staining (Periodic acid-Schiff, PAS), cyclic multiplex immunofluorescence (MxIF)) to enable mono-modality registration for the complex non-linear transformations desirable for serial section image registration. After registration, the resulting images are saved as OME-NGFF and viewed remotely via Vizarr.

The registration requires a representative $2 \mathrm{D} X Y$ plane of the data to be loaded into memory for computational image alignment using elastix as well as requiring the plane to be cast to a 32-bit floating-point data type and generation of a multi-resolution pyramid, further increasing the memory footprint when working with whole slide images. As WSIs typically contain at least 0.1 gigapixels but routinely contain 2 to 20 gigapixels of data which consume 4 bytes of memory per pixel at 32-bit floating point precision, using remote, high-performance resources is desirable to handle the large memory footprint and perform registration quickly with elastix's ${ }^{39,40}$ multi-threaded capability. Following registration, a visual check is necessary to confirm the quality of the registration at the microscopic level, and doing so remotely in the same computational environment as the registration allows rapid iteration if registration results are poor. Further, the examination of the whole slide image registrations is best done at the highest 
resolutions to see if microscopic tissue areas are aligned. Vizarr, combined with wsireg running through a remote Jupyter Notebook, allows the computation of necessary transformations and generation of pyramidal images that can be remotely visualized with all registered layers in a single view.

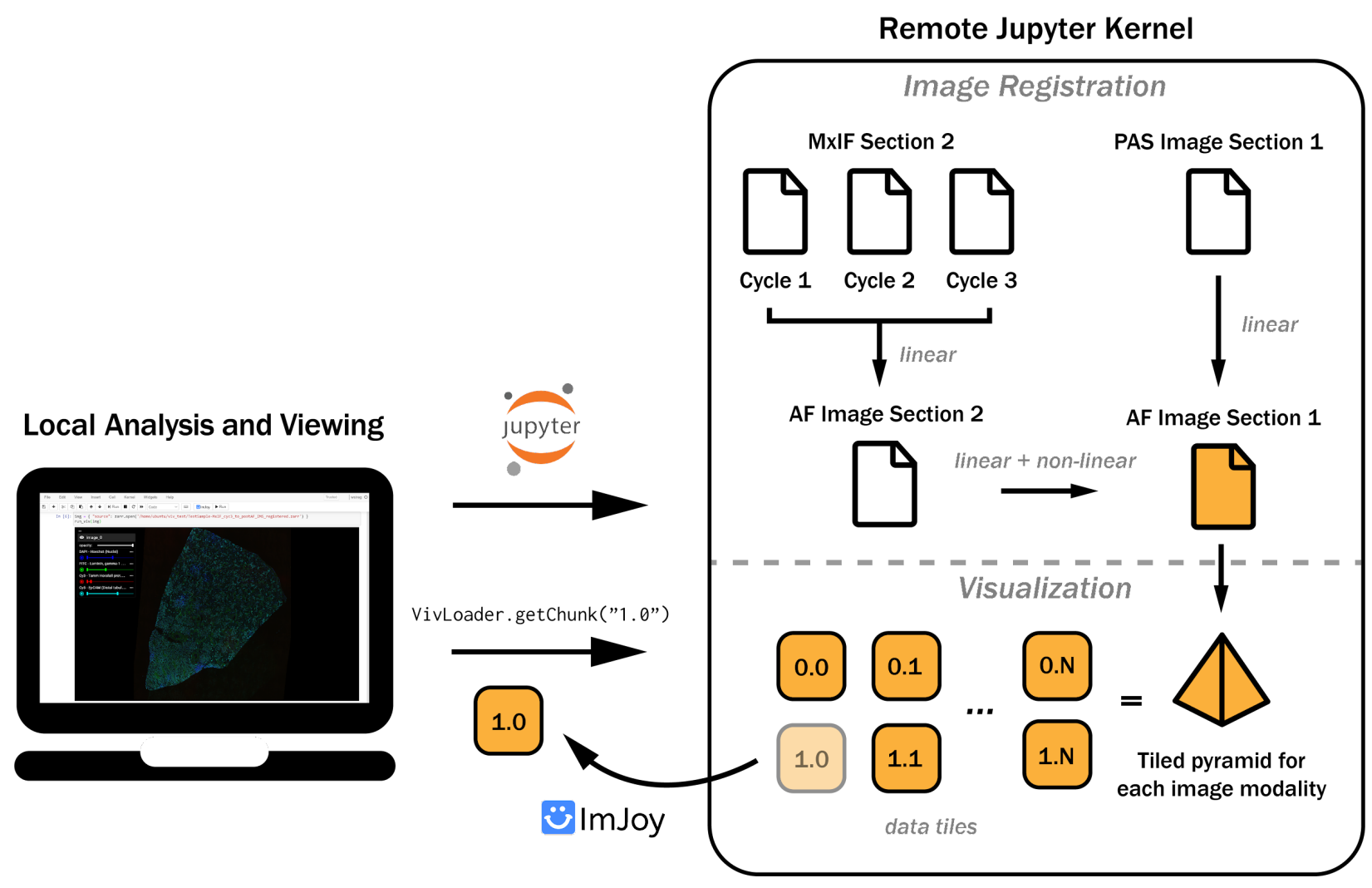

Supplementary Figure 3. Performing registration with wsireg and visualizing registration results with Vizarr through a remote Jupyter Notebook. Image registration: Images are represented by rectangles. AF Image Section 1 is the global target section and all images are resampled to the coordinate space of this image. Transformation models (linear: rigid, affine, non-linear: $b$ spline) are used for different registration paths. Linear + non-linear is used for the mono-modal serial section registration between the AF images. Remote viewing: After registration, each image is saved as OME-NGFF on the remote resources. Vizarr displays the remote data within the Jupyter Notebook. 


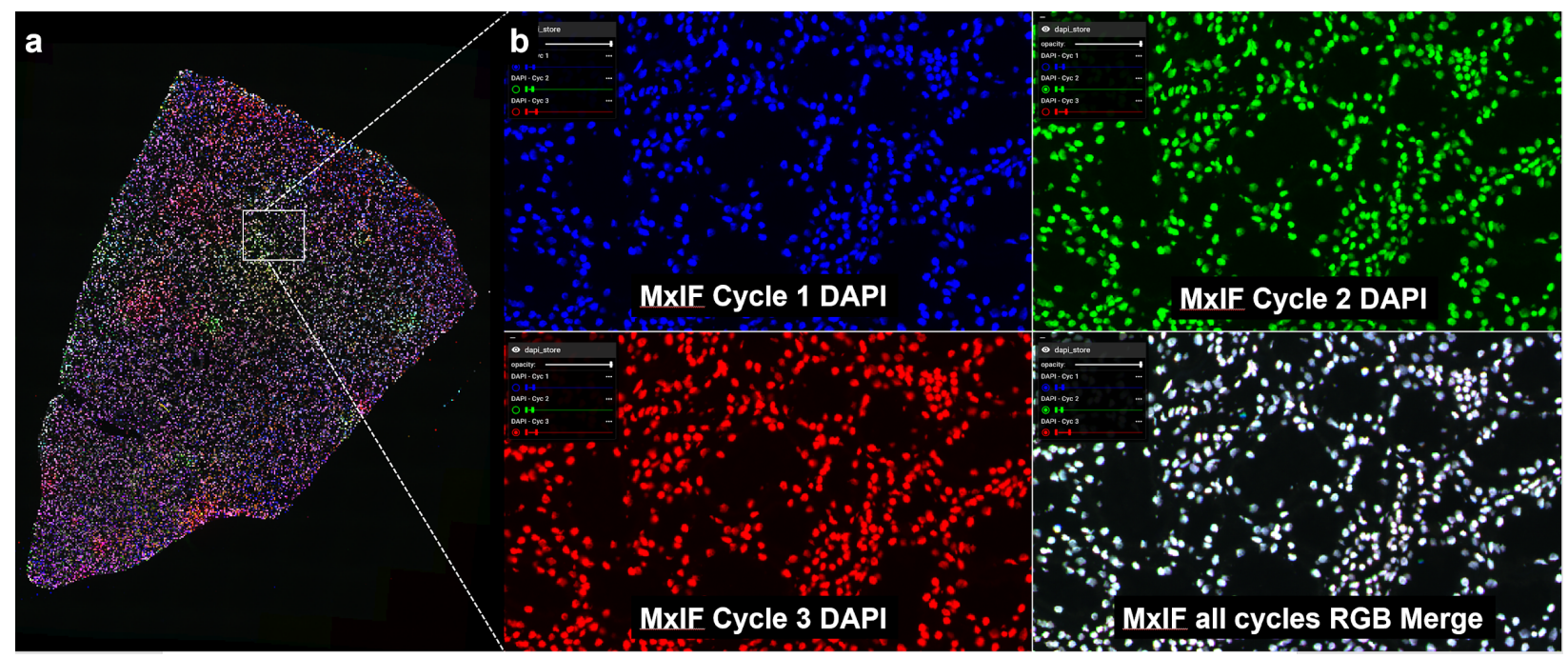

Supplementary Figure 4. Low and high-resolution views of DAPI channel (Hoechst nuclear stain) from multiplex immunofluorescence through remote Vizarr in Jupyter Notebook. (a) Low resolution "thumbnail" view of the RGB merge DAPI image from all three MxIF cycles. Some incorrect color blending is apparent at low resolution but is also due to inconsistent Hoechst intensity from the three cycles. (b) Zoomed in image to the base level of the pyramid of all three DAPI channels and the RGB merge where white indicates nucleus-nucleus overlap across all cycles.

Within the embedded viewer, different image layers can then be toggled on and off to view select subsets for quality assurance. In Supplementary Figure 4, an overlay example from cyclic multiplex immunofluorescence is demonstrated where a Hoechst nuclear stain is present in each cycle in the DAPI channel. Through Vizarr, we can visualize individual nuclei from the three registered cycle images and see their overlay, assuring that individual cells are well aligned between the cycles. Technically, this result is achieved by chunked loading of images from different datasets, showing the flexibility of Viv to handle data from multiple sources merged in a single layer. Viv, therefore, enables remote viewing of subcellular features with no compromise to spatial resolution or dynamic range of the data. 


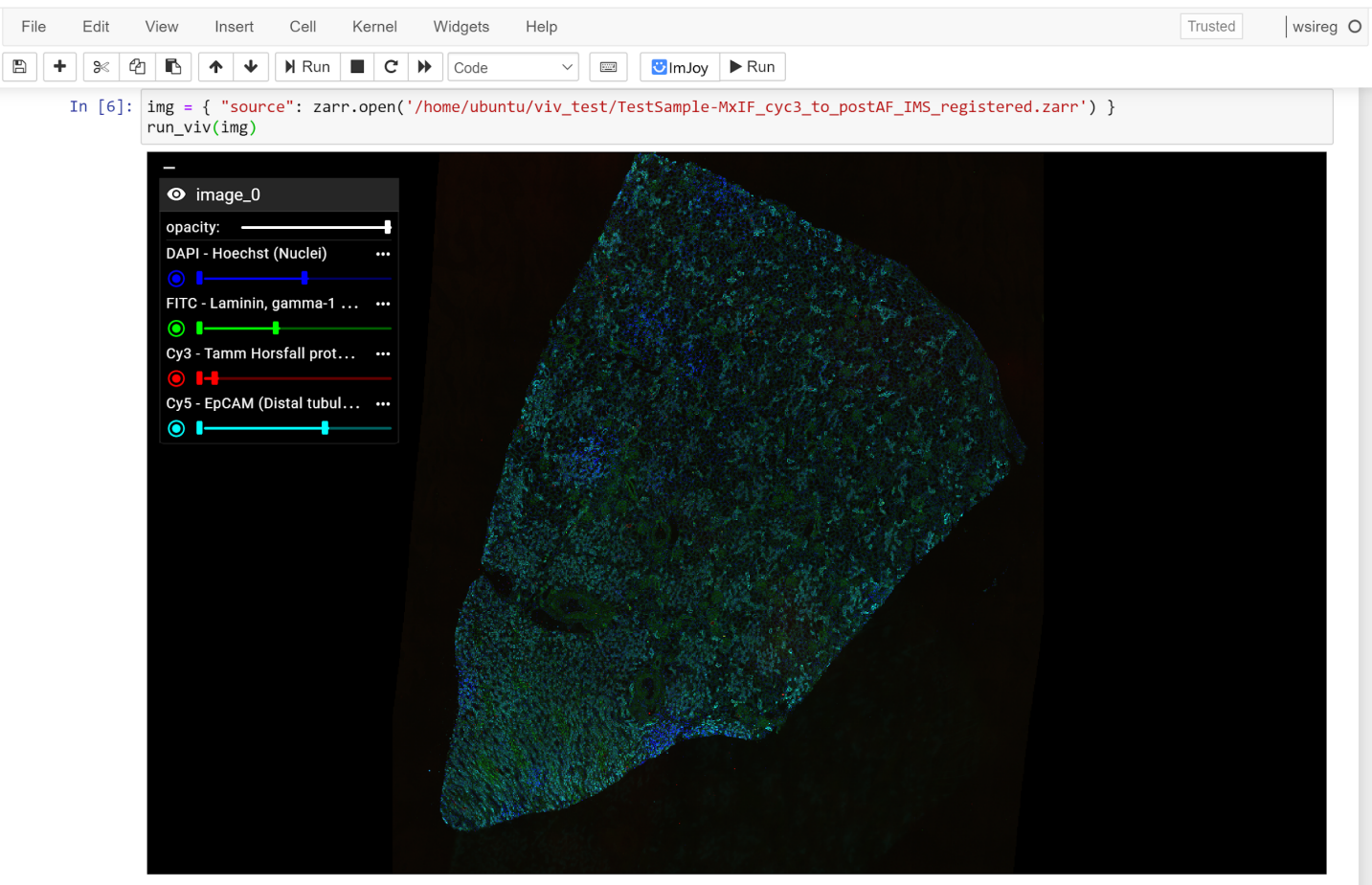

Supplementary Figure 5. Vizarr within a Juptyer Notebook. The Vizarr JavaScript implementation includes the imjoy-rpc, enabling users to programmatically view Python-based Zarr datasets within a Jupyter Notebook.

\section{Implementation}

Vizarr is a purely client-side viewer for OME-NGFF built on Viv's Zarr-based data loaders and WebGL-based rendering components. It implements a custom ImJoy ${ }^{31}$ API via the imjoy-rpc, allowing users to programmatically view large datasets stored locally on disk or in-memory NumPy arrays within a Jupyter Notebook (Supplementary Figure 5). The protocol enables secure bidirectional data exchange between the Python and JavaScript environments. The underlying key-value mapping for a Python-based Zarr object is wrapped within a custom imjoy-rpc class, allowing the Zarr JavaScript client to access chunks from any valid Python store on demand. This means that other Zarr storage backends (e.g., N5, Redis, HDF5) can be viewed using Vizarr within the Jupyter Notebook. Since the embedded viewer is entirely web-based, our plugin provides the flexibility to remotely view large datasets stored wherever a Jupyter session is running. As computational notebook environments have changed the ways that data scientists and machine learning practitioners perform and share exploratory analyses, this integration is a critical requirement for image visualization tools. Notebook formats such as Jupyter allow authors to weave code with prose and rich web-based interactive figures, forming a computational narrative ${ }^{28}$. Support for Viv in Jupyter via the ImJoy plugin allows quantitative analyses of multiplexed images to consist of code, prose, and interactive views of image channels of interest side-by-side within the same notebook. Notebooks containing code woven 
together with rich text and interactive visualizations have become essential tools for teaching, explaining, reproducing, publishing ${ }^{28}$, and open science.

Vizarr can additionally be used as a standalone web viewer for OME-NGFF ${ }^{24}$. It supports viewing both multi-resolution and high content screening datasets described under the specification. It is one of several recent interoperable tools which support OME-NGFF ${ }^{25,41}$ but is the only browser-based option.

The MIT-licensed open source code for Vizarr is available at https://github.com/hms-dbmi/vizarr. The Jupyter Notebook containing the image registration workflow and other examples are available in the same repository.

\section{Vitessce Single-Cell Visualization Framework}

Vitessce is a web-based framework for the interactive exploration of single-cell datasets ${ }^{42}$. Its flexible design allows users to author interactive visualizations which integrate epigenomics, transcriptomics, and bioimaging modalities. Visualizations are declared via JSON, specifying a responsive grid layout of coordinated multiple views. Views may be coordinated via synchronized interactions (e.g., zoom and pan) or linked through biological entities such as cells or genes. The Human Biomolecular Atlas Program (HuBMAP) Data Portal embeds Vitessce to visualize datasets derived from multi-modal and spatially-resolved assays, such as CO-Detection by indEXing (CODEX), autofluorescence microscopy, and MALDI imaging mass spectrometry.

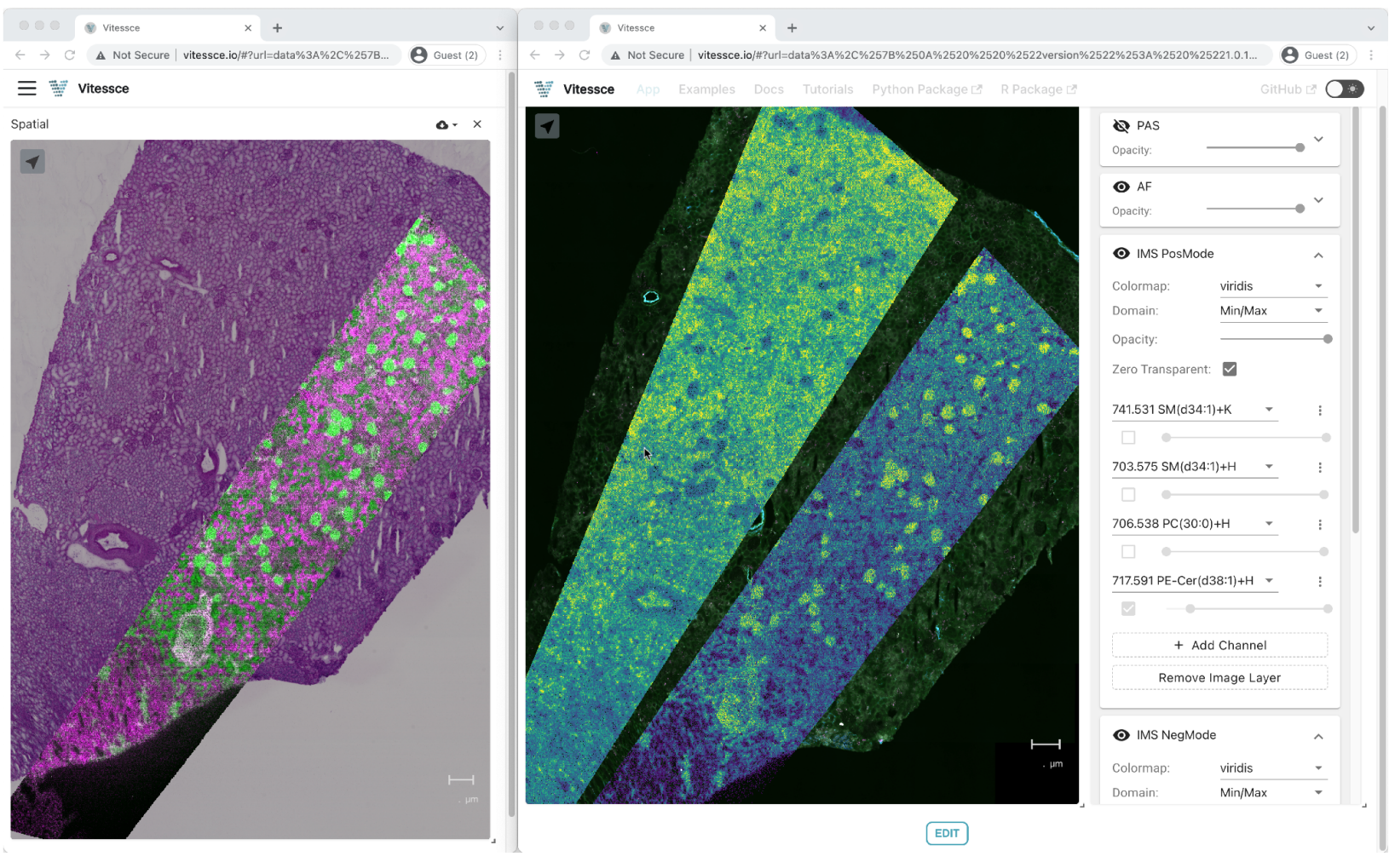


Supplementary Figure 6. Vitessce orchestrates the stacking of multiple imaging modalities in a single view. The imaging datasets shown comprise PAS (periodic-acid Schiff stain) microscopy, autofluorescence microscopy, and MALDI imaging mass spectrometry experiments, capturing various spatial resolutions and regions of the target tissue. Spatial transformations are defined in a top-level configuration per modality and applied by Viv on the GPU, and controls in the user interface adjust channel visibility, opacity, and color mapping through Viv's API.

The spatial visualization component of Vitessce renders bioimaging datasets using Viv. Affine transformations, defined in a Vitessce configuration, are applied by Viv on the GPU and allow imaging datasets captured from various assays, spatial regions, and resolutions to be overlaid in the same coordinate space (Supplementary Figure 6). The Vitessce user interface exposes controls to modify the opacity, channel visibility, and color mappings for individual image layers through Viv's API. Centrally, Vitessce composes Viv image layers with other layers for non raster-based data (e.g., cell segmentation overlays, spatially-resolved gene expression) to enable simultaneous visual exploration of multiple data modalities (Supplementary Figure 7).

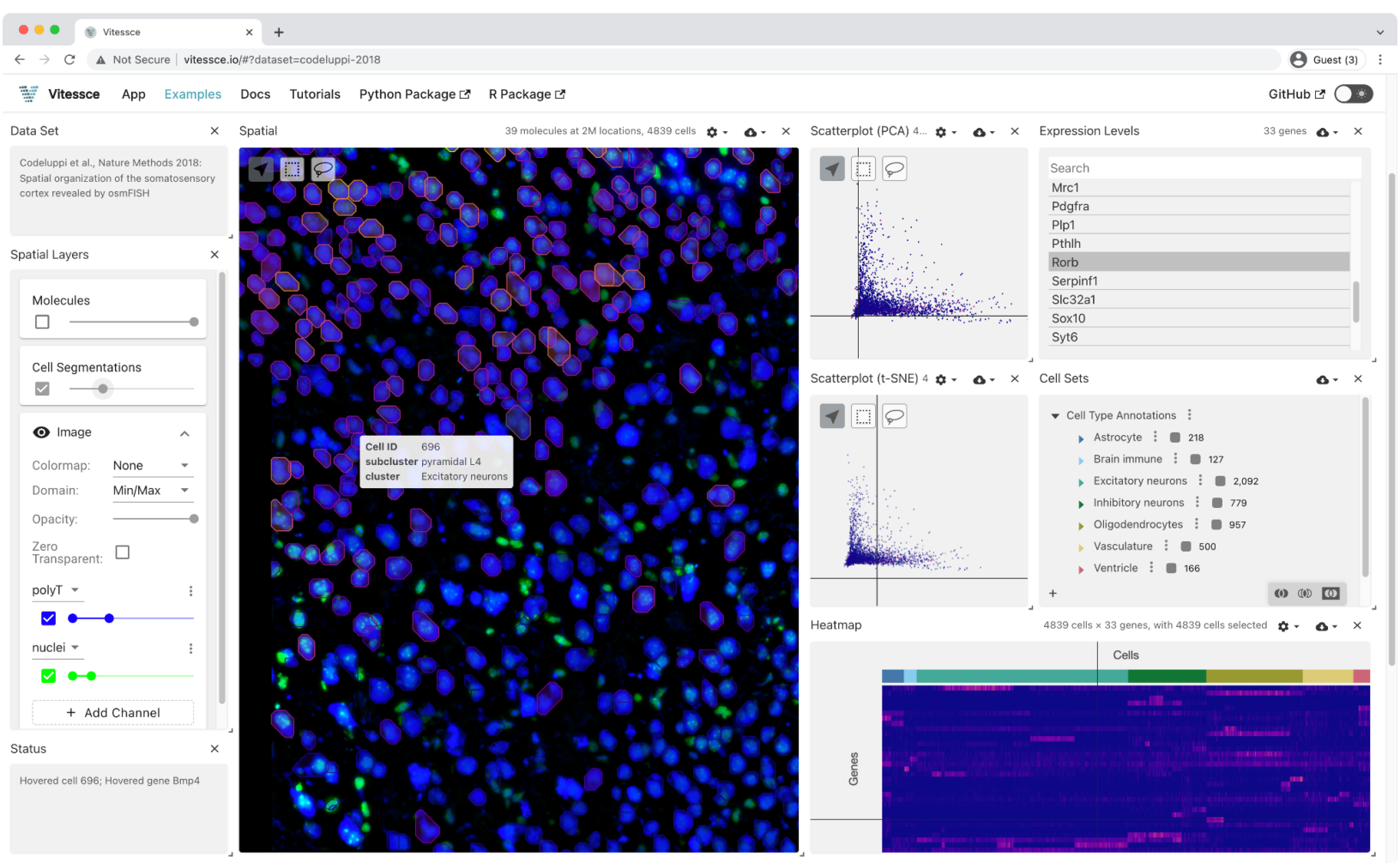

Supplementary Figure 7. Vitessce overlays spatially-resolved gene expression on a fluorescence microscopy dataset captured from mouse somatosensory cortex ${ }^{43}$. Cell segmentation polygons are colored by relative gene expression and stacked over Viv's imaging layers in the Spatial view component. Gene selection is coordinated by Vitessce across multiple linked views, and users are able to toggle the display of individual spatial layers as well as adjust the rendering parameters of the Viv-based imaging layers. 


\section{Implementation}

Vitessce combines multiple views in a singular React-based user interface, and interactions are synced across views via a coordinated multiple view implementation. Vitessce is written in JavaScript and requires Viv as a dependency to support loading, transforming, and rendering large bioimaging datasets. Vitessce additionally implements client-side data loaders for common single-cell formats, like AnnData ${ }^{44}$, allowing a flexible integration with computational analysis tools and data resources. Widgets for Python and $\mathrm{R}$ are available for creating and interacting with Vitessce visualizations in computational notebook environments.

The MIT-licensed source code for Vitessce is available at https://github.com/vitessce/vitessce.

\section{Avivator Image Viewer}

We created Avivator (http://avivator.gehlenborglab.org), a JavaScript and React-based image viewer that displays OME-TIFF and Zarr datasets directly from a user-provided remote URL. Avivator also supports viewing local files without a web-server via the file picker in the user interface.

Avivator implements continuous zooming and panning and showcases the advantages of client-side image rendering. A slider interface allows users to modify the channel intensities and the image updates as soon as the sliders are being dragged. As the updates are instantaneous due to client-side image rendering, this approach is efficient for finding adequate intensity settings. Avivator implements both additive blending and additive color mapping. 


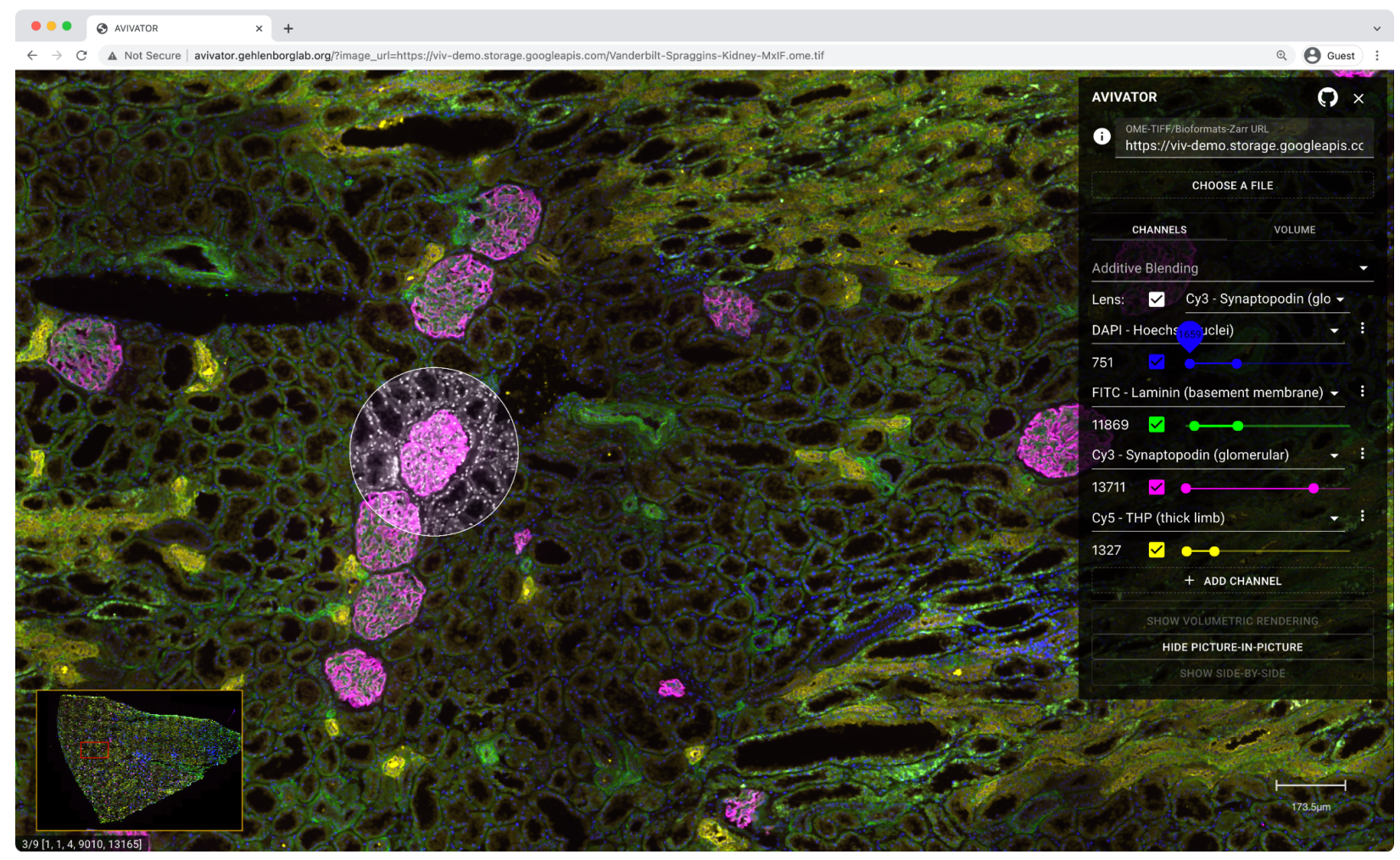

Supplementary Figure 8. Avivator rendering a pyramidal, multiplexed immunofluorescence OME-TIFF image of a human kidney using additive blending to render four image channels into a single RGB image in the client. The picture-in-picture view in the lower left corner provides context. A magic lens applying a local transformation of the image data to emphasize the "Cy3 Synaptopodin" channel.

Furthermore, Avivator uses magic lenses ${ }^{45}$ that locally re-render the image with different transformation settings, e.g., to highlight a particular image channel (see Supplementary Figure 8). As all operations take place in the client, there is no noticeable lag when moving the lens over the image. Scale bars as well as a picture-in-picture (see Supplementary Figure 8) and linked side-by-side views (see Supplementary Figure 10) that enable the overview + detail visualization paradigm are also supported. Image annotations are beyond the scope of this Viv use case but can be added to Avivator by integrating vector layers provided by Deck.gl. 


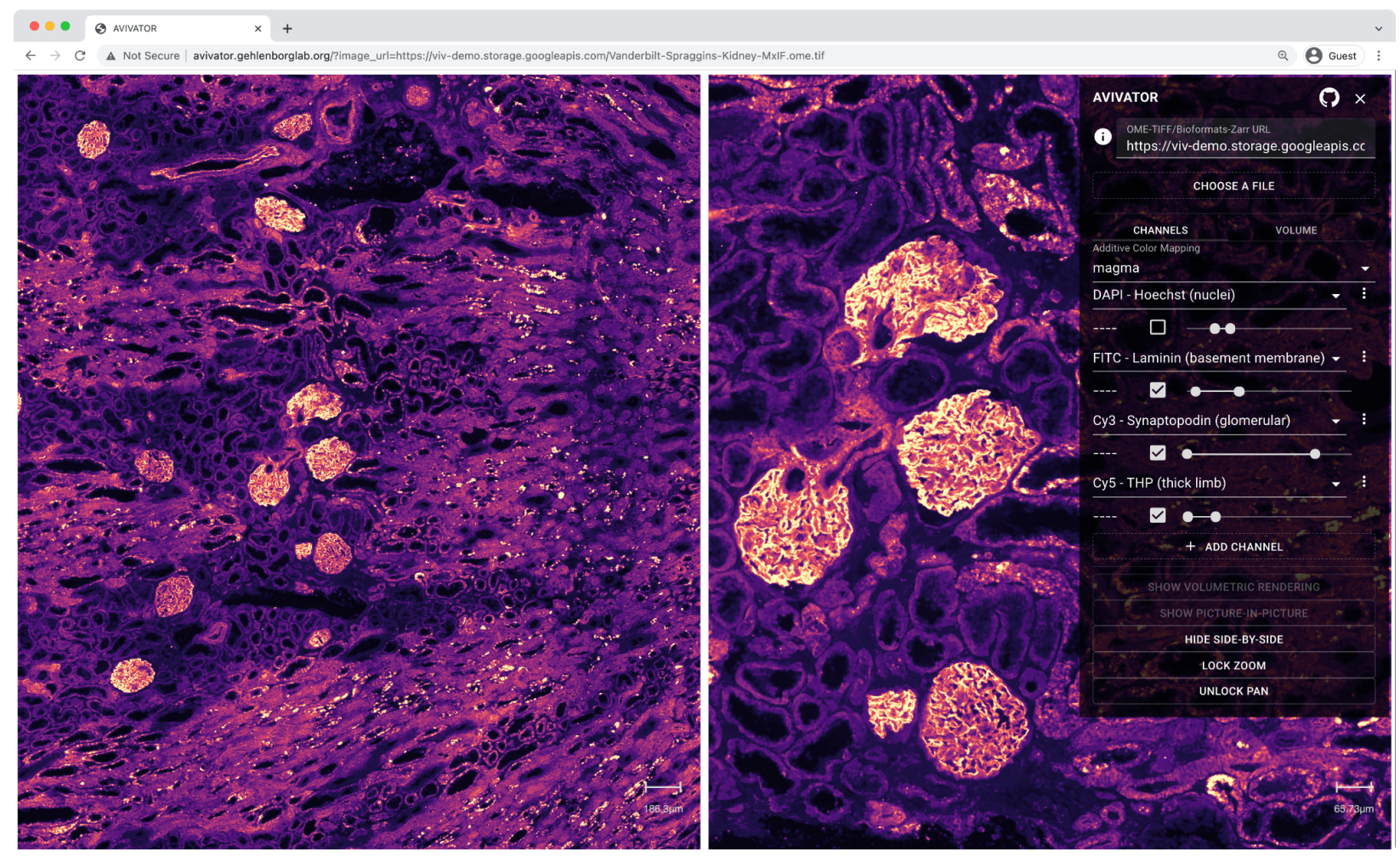

Supplementary Figure 9. User interface of Avivator rendering a pyramidal multiplexed immunofluorescence OME-TIFF image of a human kidney as two linked views with a detail view on the left and an overview on the right. Three active channels (FITC, Cy3, Cy5) are mapped to the "magma" color map using the additive color mapping approach built into Viv.

Avivator also exposes the raycasting implemented in Viv, rendering 3D volumes of datasets with $Z$ stacks using either maximum intensity projection, minimum intensity projection, or additive blending (see Supplementary Figure 10). Unlike multiscale 2D rendering, which dynamically retrieves data chunks from pyramidal sub-resolutions, Viv needs to load the full $Z$ stack for the selected channels in order to render the volume. In Avivator, the user selects at which downsampled resolution the volume should be rendered, and Viv retrieves the $Z$ stack corresponding to this selection. Channel selection, dynamic color mapping, and clipping planes along the $\mathrm{X}, \mathrm{Y}$, and $\mathrm{Z}$ axis are supported in the user interface. 


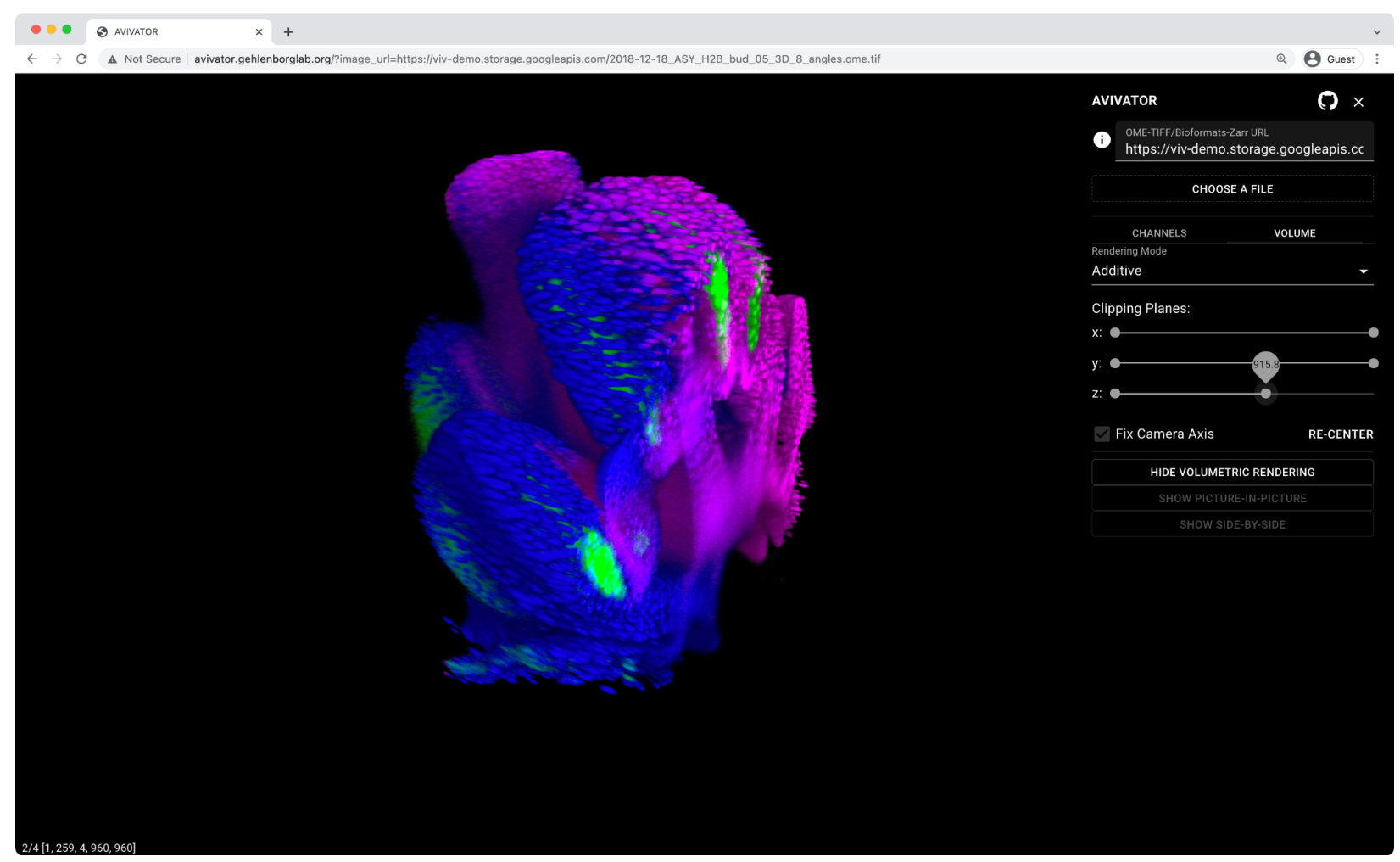

Supplementary Figure 10. Avivator displaying a volumetric rendering of an OME-TIFF with a $Z$-stack via Viv-based raycasting. A clipping plane is adjusted along the $Z$ axis to display an orthogonal slice of the volume.

Since datasets are directly visualized by Avivator, users can directly download the source dataset for further analysis by copying the data URL in the browser address bar. This compatibility of image formats shared by Viv and the rest of the image analysis ecosystem illustrates a powerful mechanism for users to share data internally, with collaborators, or the broader scientific community.

Users can upload primary data to persistent cloud object storage or local web servers and provide a URL in a client application to view these resources, eliminating the need to pre-render or configure sophisticated server-client architectures, while providing the option to download and analyze with other tools. Cloud object storage is becoming increasingly popular for storing archival data for growing image resources, such as the European Bioinformatics Institute (EBI)-based Image Data Resource (IDR) ${ }^{28}$, and having client software that can visualize the data in the resources directly is essential to making these resources accessible at scale.

\section{Implementation}

Avivator is an open source React application that combines multiple Viv layers and views, which are extensions of Deck.gl. Its source code is available as part of Viv at https://github.com/hms-dbmi/viv and at https://www.npmjs.com/package/@hms-dbmi/viv. A 
collection of sample images are provided on commercial cloud storage and accessible directly via URL in the web application.

\section{Supplementary References}

1. Aeffner, F. et al. Introduction to Digital Image Analysis in Whole-slide Imaging: A White Paper from the Digital Pathology Association. J. Pathol. Inform. 10, 9 (2019).

2. Rashid, R. et al. Interpretative guides for interacting with tissue atlas and digital pathology data using the Minerva browser. bioRxiv 2020.03.27.001834 (2020) doi:10.1101/2020.03.27.001834.

3. Krueger, R. et al. Facetto: Combining Unsupervised and Supervised Learning for Hierarchical Phenotype Analysis in Multi-Channel Image Data. IEEE Trans. Vis. Comput. Graph. 26, 227-237 (2020).

4. Uhlen, M. et al. Towards a knowledge-based Human Protein Atlas. Nat. Biotechnol. 28, 1248-1250 (2010).

5. Allan, C. et al. OMERO: flexible, model-driven data management for experimental biology. Nat. Methods 9, 245-253 (2012).

6. Gutman, D. A. et al. The Digital Slide Archive: A Software Platform for Management, Integration, and Analysis of Histology for Cancer Research. Cancer Res. 77, e75-e78 (2017).

7. Rubens, U. et al. Cytomine: Toward an Open and Collaborative Software Platform for Digital Pathology Bridged to Molecular Investigations. Proteomics Clin. Appl. 13, e1800057 (2019).

8. OpenSeadragon. https://openseadragon.github.io/.

9. Gratier, T., Spencer, P. \& Hazzard, E. OpenLayers 3 : Beginner's Guide. (Packt Publishing Ltd, 2015). 
10. Goltsev, Y. et al. Deep Profiling of Mouse Splenic Architecture with CODEX Multiplexed Imaging. Cell 174, 968-981.e15 (2018).

11. Rashid, R. et al. Highly multiplexed immunofluorescence images and single-cell data of immune markers in tonsil and lung cancer. Sci Data 6, 323 (2019).

12. Ijsselsteijn, M. E., van der Breggen, R., Farina Sarasqueta, A., Koning, F. \& de Miranda, N. F. C. C. A 40-Marker Panel for High Dimensional Characterization of Cancer Immune Microenvironments by Imaging Mass Cytometry. Front. Immunol. 10, 2534 (2019).

13. Lin, J.-R., Fallahi-Sichani, M. \& Sorger, P. K. Highly multiplexed imaging of single cells using a high-throughput cyclic immunofluorescence method. Nat. Commun. 6, 8390 (2015).

14. Neumann, E. K., Djambazova, K., Caprioli, R. M. \& Spraggins, J. M. Multimodal Imaging Mass Spectrometry: Next Generation Molecular Mapping in Biology and Medicine. J. Am. Soc. Mass Spectrom. (2020) doi:10.1021/jasms.0c00232.

15. Moore, J. et al. OMERO and Bio-Formats 5: flexible access to large bioimaging datasets at scale. in vol. 9413941307 (International Society for Optics and Photonics, 2015).

16. itk-vtk-viewer. itk-vtk-viewer https://kitware.github.io/itk-vtk-viewer/index.html.

17. neuroglancer. https://github.com/google/neuroglancer.

18. Boergens, K. M. et al. webKnossos: efficient online 3D data annotation for connectomics. Nat. Methods 14, 691-694 (2017).

19. Saalfeld, S., Cardona, A., Hartenstein, V. \& Tomancak, P. CATMAID: collaborative annotation toolkit for massive amounts of image data. Bioinformatics 25, 1984-1986 (2009).

20. Miles, A. et al. zarr-developers/zarr-python: v2.4.0. (2020). doi:10.5281/zenodo.3773450.

21. Wang, Y. Deck. gl: Large-scale web-based visual analytics made easy. arXiv preprint arXiv:1910.08865 (2019). 
22. Alted, F. Blosc, an extremely fast, multi-threaded, meta-compressor library. https://blosc.org/.

23. Goldberg, I. G. et al. The Open Microscopy Environment (OME) Data Model and XML file: open tools for informatics and quantitative analysis in biological imaging. Genome Biol. 6, R47 (2005).

24. Moore, J. et al. OME-NGFF: a next-generation file format for expanding bioimaging data-access strategies. Nat. Methods 1-3 (2021).

25. Sofroniew, N. et al. napari/napari: 0.2.8. (2019). doi:10.5281/zenodo.3592005.

26. Schindelin, J. et al. Fiji: an open-source platform for biological-image analysis. Nat. Methods 9, 676-682 (2012).

27. Bankhead, P. et al. QuPath: Open source software for digital pathology image analysis. Sci. Rep. 7, 16878 (2017).

28. Williams, E. et al. The Image Data Resource: A Bioimage Data Integration and Publication Platform. Nat. Methods 14, 775-781 (2017).

29. Consortium, H. \& HuBMAP Consortium. The human body at cellular resolution: the NIH Human Biomolecular Atlas Program. Nature vol. 574 187-192 (2019).

30. Dekker, J. et al. 4D Nucleome Network. Corrigendum: The 4D nucleome project. Nature 552, $278(2017)$.

31. Ouyang, W., Mueller, F., Hjelmare, M., Lundberg, E. \& Zimmer, C. ImJoy: an open-source computational platform for the deep learning era. Nat. Methods 16, 1199-1200 (2019).

32. Welch, T. A. A technique for high-performance data compression. Computer 8-19 (1984).

33. WebGL Insights. (A K Peters/CRC Press, 2015).

34. Li, H. Tabix: fast retrieval of sequence features from generic TAB-delimited files. Bioinformatics 27, 718-719 (2011). 
35. Li, H. et al. The Sequence Alignment/Map format and SAMtools. Bioinformatics 25, 2078-2079 (2009).

36. Valuchova, S. et al. Imaging plant germline differentiation within Arabidopsis flowers by light sheet microscopy. Elife 9, e52546 (2020).

37. Patterson, N. H., Tuck, M., Van de Plas, R. \& Caprioli, R. M. Advanced Registration and Analysis of MALDI Imaging Mass Spectrometry Measurements through Autofluorescence Microscopy. Anal. Chem. 90, 12395-12403 (2018).

38. Jones, M. A. et al. Discovering New Lipidomic Features Using Cell Type Specific Fluorophore Expression to Provide Spatial and Biological Specificity in a Multimodal Workflow with MALDI Imaging Mass Spectrometry. Anal. Chem. 92, 7079-7086 (2020).

39. Klein, S., Staring, M., Murphy, K., Viergever, M. A. \& Pluim, J. P. W. elastix: a toolbox for intensity-based medical image registration. IEEE Trans. Med. Imaging 29, 196-205 (2010).

40. Marstal, K., Berendsen, F., Staring, M. \& Klein, S. SimpleElastix: A User-Friendly, Multi-lingual Library for Medical Image Registration. in 2016 IEEE Conference on Computer Vision and Pattern Recognition Workshops (CVPRW) 574-582 (2016).

41. Vergara, H. M. et al. Whole-body integration of gene expression and single-cell morphology. Cell 184, 4819-4837.e22 (2021).

42. Keller, M. S. et al. Vitessce: a framework for integrative visualization of multi-modal and spatially-resolved single-cell data. OSF Preprints (2021) doi:10.31219/osf.io/y8thv.

43. Codeluppi, S. et al. Spatial organization of the somatosensory cortex revealed by osmFISH. Nat. Methods 15, 932-935 (2018).

44. Wolf, F. A., Angerer, P. \& Theis, F. J. SCANPY: large-scale single-cell gene expression data analysis. Genome Biol. 19, 15 (2018).

45. Bier, E. A., Stone, M. C., Pier, K., Buxton, W. \& DeRose, T. D. Toolglass and magic lenses: 
the see-through interface. in 73-80 (ACM Press, 1993). 